\title{
Longitudinal change in autonomic symptoms predicts activities of daily living and depression in Parkinson's disease
}

\author{
Miriam Sklerov ${ }^{1}$ (D) Chia-Hao Shih ${ }^{2} \cdot$ Nina Browner $^{1} \cdot$ Jose-Alberto Palma $^{3} \cdot$ Martin Styner $^{4} \cdot$ Eran Dayan $^{5,6}$
}

Received: 24 November 2019 / Accepted: 7 February 2020 / Published online: 20 February 2020

(c) Springer-Verlag GmbH Germany, part of Springer Nature 2020

\begin{abstract}
Purpose The primary objective of this study was to examine the relationship of longitudinal changes in autonomic symptom burden and longitudinal changes in activities of daily living (ADLs); a secondary analysis examined the impact of depressive symptoms in this relationship.

Methods Data were retrieved from the Parkinson's Progression Markers Initiative (PPMI), a dataset documenting the natural history of newly diagnosed Parkinson's disease (PD). The analysis focused on data from baseline, visit 6 (24 months after enrollment), and visit 12 (60 months after enrollment). The impact of longitudinal changes in autonomic symptom burden on longitudinal changes in ADLs function was examined. A secondary mediation analysis was performed to investigate whether longitudinal changes in depressive symptoms mediate the relationship between longitudinal changes in autonomic symptom burden and ADLs function.

Results Changes in autonomic symptom burden, cognitive function, depressive symptoms, and motor function all correlated with ADLs. Only changes in ADLs and depression were found to be associated with changes in autonomic symptom burden. We found that longitudinal change in autonomic symptoms was a significant predictor of change in ADLs at 24 and 60 months after enrollment, with the cardiovascular subscore being a major driver of this association. Mediation analysis revealed that the association between autonomic symptoms and ADLs is partially mediated by depressive symptoms.

Conclusions Longitudinal changes in autonomic symptoms impact ADLs function in patients with early signs of PD, both directly and indirectly through their impact on depressive symptoms. Future investigation into the influence of treatment of these symptoms on outcomes in PD is warranted.
\end{abstract}

Keywords Activities of daily living · Autonomic symptoms · Depressive symptoms · Parkinson's disease

Electronic supplementary material The online version of this article (https://doi.org/10.1007/s10286-020-00672-7) contains supplementary material, which is available to authorized users.

Miriam Sklerov

sklerovm@neurology.unc.edu

Chia-Hao Shih

chiahao.shih@utoledo.edu

Nina Browner

brownern@neurology.unc.edu

Jose-Alberto Palma

josealberto.palmacarazo@nyulangone.org

Martin Styner

styner@email.unc.edu

Eran Dayan

eran_dayan@med.unc.edu

1 Department of Neurology, University of North Carolina, 170

Manning Drive, CB\# 7025, Chapel Hill, NC 27599, USA
2 Department of Psychiatry, University of Toledo, Ruppert Health Center 0004, Mail Stop 1193, 3000 Arlington Ave., Toledo, OH 43614-2598, USA

3 Department of Neurology, Dysautonomia Center, New York University Medical Center, 530 First Avenue, Suite 9Q, New York, NY 10016, USA

4 Department of Psychiatry, University of North Carolina, 352 Medical School Wing C, Chapel Hill, NC 27516, USA

5 Biomedical Research Imaging Center, University of North Carolina, 130 Mason Farm Road, CB\# 7513, Chapel Hill, NC 27599, USA

6 Department of Radiology, University of North Carolina, 130 Mason Farm Road, CB\# 7513, Chapel Hill, NC 27599, USA 


\section{Introduction}

Parkinson's disease (PD) is characterized by motor and non-motor symptoms [1]. There is broad variability in clinical phenotypes and rates of progression in this disease [2-4]. How to predict outcomes for individuals with PD remains an important question, with healthcare and clinical research implications.

Many factors have been identified as influential in predicting outcomes in PD. Frequently investigated prognostic factors in PD include age, disease duration, depression, gait impairment, and PD motor subtype [3-5]. Autonomic dysfunction is common in persons with PD and strongly impacts prognosis [6, 7]. Most studies investigating prognostic predictors of PD have focused on cross-sectional data $[2-5,8]$. Few studies have investigated the impacts of longitudinal changes in autonomic symptoms over time on functional decline in PD patients [9, 10], and none, to our knowledge, have been performed in a homogenous cohort of patients diagnosed with early stage PD over an extended period of time. This presents a crucial gap in knowledge as longitudinal changes in some clinical factors have been found to influence decline in health-related quality of life $[5,11]$.

We hypothesized that an increasing burden of autonomic symptoms over time is negatively associated with changes in activities in daily living (ADLs) function in PD. Additionally, we hypothesized that depression would emerge as a driver of the change in ADLs function over time, as this has been demonstrated previously in different PD populations $[12,13]$. With this aim we evaluated the impact and relationships of longitudinal changes in autonomic symptom burden on changes in ADLs and depression scores in a prospective cohort of patients with early stage PD over a period of 2 and 5 years.

\section{Methods}

\section{Subjects}

The data for these analyses were obtained from the Parkinson's Progression Marker Initiative (PPMI), a publiclyavailable, longitudinal, prospective database of over 400 patients with early stage PD [14]. Full information about the PPMI study, including inclusion/exclusion criteria, recruitment, sites, full list of evaluations and procedures, can be found at www.ppmi-info.org. Subjects underwent regular standardized and validated evaluations, clinical and cognitive assessments, imaging examinations, and biological sampling. Inclusion criteria requirements included symptoms of rest tremor, bradykinesia, or rigidity, and a diagnosis of PD for $<2$ years at screening. Eligible subjects were at Hoehn and Yahr stage I or II at enrollment, had neuroimaging findings on dopamine transporter single-photon emission computed tomography (DaT SPECT) or positron-emission tomography examinations that supported the PD diagnosis, and were not taking medications for PD within 6 months of the baseline visit.

Of the over 400 currently enrolled subjects in PPMI, subject data were chosen for inclusion in the current analysis based on the presence of complete data at visit 6 (24 months from baseline, $N=266$ ), and at visit 12 (60 months from baseline, $N=226$ ).

All research procedures included in PPMI were approved by the local Institutional Review Boards of the participating centers. All participants provided written informed consent before enrollment into the study [14].

\section{Clinical Evaluations and Scales}

All evaluations and scales used in this analysis were collected as part of the PPMI protocol. Autonomic symptom burden was evaluated using the Scales for Outcomes in Parkinson's disease-Autonomic (SCOPA-AUT) [15], a validated questionnaire of autonomic symptoms. The SCOPA-AUT includes seven questions about gastrointestinal function, six questions about urinary function, three questions about cardiovascular function, four questions about thermoregulatory function, one question about pupillomotor function, and three questions about sexual function. Higher scores indicate higher burden of autonomic symptoms (score range 0-69 points).

Motor function was evaluated using the Movement Disorders Society Unified Parkinson's Disease Rating Scale (MDS-UPDRS) [16] part III motor score, an objective examination performed by a trained evaluator. All MDSUPDRS part III scores included in the current study were in the "off" medication state. A higher MDS-UPDRS score (range 7-86 points) indicates a higher degree of motor dysfunction. ADLs function was evaluated with the Schwab and England (S\&E) Activities of Daily Living (ADLs) scale, in which a higher value indicates better function (score range 10-100\%). No additional measures of ADLs function or measures of quality of life were included in the PPMI study. Depression was measured using the Geriatric Depression Scale (GDS), in which higher score indicates a higher burden of depressive symptoms (score range 0-15 points) [17]. Cognitive function was assessed using the Montreal Cognitive Assessment (MoCA) screening tool, in which a lower score indicates worse cognitive function [18]. 


\section{Outcome Measures and Statistical Analysis}

The primary independent variable in this analysis was the change in SCOPA-AUT score from baseline to visit 6 (month 24), and from baseline to visit 12 (month 60), and the primary dependent variable was change in S\&E score over this same time period. Secondary endpoints were longitudinal changes in the GDS [17], MoCA [18], and the MDS-UPDRS part III [16]. Orthostatic hypotension (OH) was defined as a reduction in blood pressure of $20 \mathrm{mmHg}$ systolic, or $10 \mathrm{mmHg}$ diastolic, with changing from the supine to standing position, as reported in the PPMI database. Statistical analyses were performed using SAS 9.4 statistical software (SAS Institute Inc., Cary, NC, USA) and the PROCESS macro developed by Hayes [19]. Means, standard deviations, and ranges were obtained for demographic variables and for all scales included in the analysis (Table 1). Two-sample $t$ tests were used to compare means of continuous clinical variables at different visits, and the Chi-square test was performed to compare categorical variables in different visits. The Spearman correlation coefficient was used to evaluate associations between clinical variables. Simple linear regression analysis was utilized to evaluate the impact of longitudinal changes in SCOPA-AUT on longitudinal changes in ADLs scores. Mediation analysis was performed to evaluate whether depression, as measured by the GDS, may mediate the association between autonomic symptom burden, as measured by the SCOPA-AUT, and ability to perform ADLs, as measured by the S\&E scale. In order to test the statistical significance of the mediation, or indirect effects, $95 \%$ confidence intervals (CIs) were established by using a bootstrapping approach with 5000 samples. Statistical significance in these tests was obtained whenever the $95 \%$ CI fell outside the value of zero. To test whether this mediation model was moderated by severity of motor symptoms in this cohort, a moderated mediation model was used to investigate whether the strength and/or direction of the proposed indirect effect of depressive symptoms was contingent upon subject's severity of motor dysfunction, as assessed by MDS-UPDRS part III scores.

\section{Results}

We analyzed data from all 266 subjects in the PPMI database who had data available for the clinical variables of interest at visit 6 (24 months), and all 226 subjects who had data available for the clinical variables of interest at visit 12 (60 months). Between baseline and visit 6 , and baseline and visit 12 , there was a decline in the S\&E, SCOPA-AUT, MoCA, GDS, and MDS-UPDRS III scores (Table 1). Demographic data and descriptive statistics for the clinical variables are summarized in Table 1.

Longitudinal increases in the SCOPA-AUT score from baseline were significantly associated with worsening S\&E score at visit $12(r=-0.170, p=0.010)$ but not visit 6 $(r=-0.095, p=0.124)$ (Fig. 1). Increasing SCOPA-AUT score was also associated with increasing GDS score at visit $6(r=0.203, p=0.001)$ but not at visit $12(r=0.126$, $p=0.058)$. Correlations between S\&E, SCOPA-AUT, GDS, MDS-UPDRS part III, and MoCA scores, and age at visit 6 and visit 12 are presented in Fig. 1.
Table 1 Demographics and clinical variables of subjects with Parkinson's disease at baseline, visit 6 , and visit 12

\begin{tabular}{llll}
\hline Demographics and clinical variables & Baseline $(N=315)$ & $\begin{array}{l}\text { Visit 6 (24 months } \\
\text { from baseline) } \\
(N=266)\end{array}$ & $\begin{array}{l}\text { Visit 12 (60 months } \\
\text { from baseline) } \\
(N=226)\end{array}$ \\
\hline Male, $N(\%)$ & $230(73.0 \%)$ & $180(67.7 \%)$ & $152(67.3 \%)$ \\
Age (years) & $62.5(9.8)$ & & \\
Disease duration (years) & $0.6(0.7)$ & $88.5(7.7)^{* *}$ & $84.93(10.0)^{* *}$ \\
S\&E ADLs scale score & $92.8(5.8)$ & $11.3(6.9)^{* *}$ & $13.1(7.9)^{* *}$ \\
SCOPA-AUT score & $9.3(6.1)$ & $26.0(3.2)^{* *}$ & $26.6(3.3)^{* *}$ \\
MoCA scale score & $26.9(2.4)$ & $27.4(11.5)^{* *}$ & $31.0(11.8)^{* *}$ \\
MDS-UPDRS part III score & $21.2(8.9)$ & $2.7(2.8)^{* *}$ & $2.7(2.7)^{* *}$ \\
GDS score & $2.4(2.7)$ & $1.8(0.6)^{* *}$ & $2.0(0.4)^{*}$ \\
Hoehn and Yahr stage & $1.5(0.5)$ & & \\
\hline
\end{tabular}

All values are presented as the mean with the standard deviation (SD) in parenthesis, with the exception of number $(\%)$ of males in study

GDS Geriatric Depression Scale, MoCA Montreal Cognitive Assessment, MDS-UPDRS 3 Movement Disorders Society Unified Parkinson's Disease Rating Scale part 3 (motor examination), $S \& E A D L s$ Schwab and England Activities of Daily Living, SCOPA-AUT Scale for Outcomes in Parkinson's disease for Autonomic Symptoms

${ }^{*} p<0.01$ difference between this value and baseline value; ${ }^{* *} p<0.001$ difference between this value and baseline value 


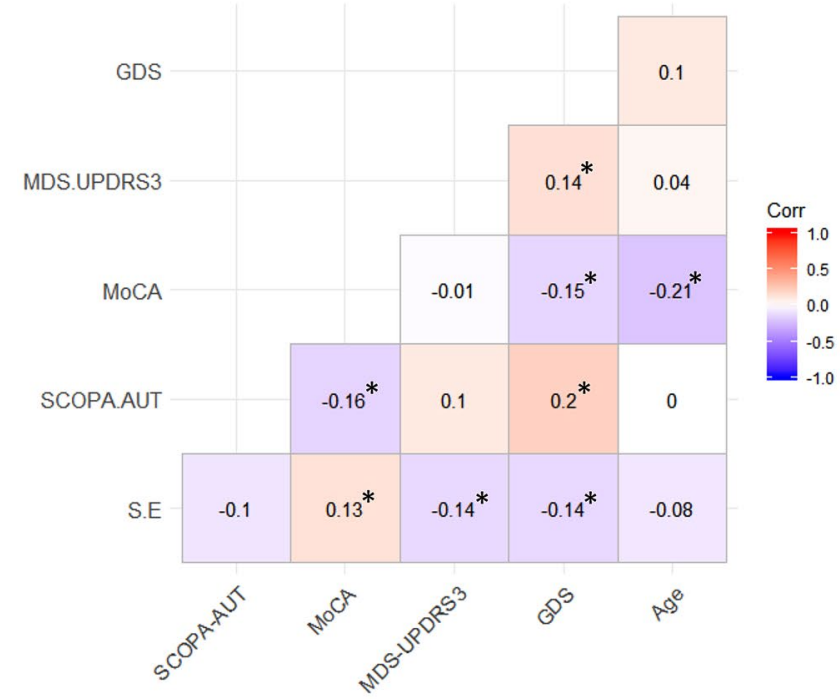

Fig. 1 Associations of longitudinal changes in clinical variables between baseline and visit 6 (a; 24 months from baseline), and between baseline and visit 12 (b; 60 months from baseline). Values are expressed as the Spearman correlation coefficient. Asterisk indicates a signficant difference at $p<0.05$. GDSGeriatric Depression

We then investigated whether taking medication for depression or medications that lower blood pressure could influence our results. Subjects were categorized as "taking" or "not taking" medications for depression or medications that lower blood pressure, respectively. There was a significant association between depression medication utilization and SCOPA-AUT score at baseline, visit 6 , and visit $12(r=0.196, p=0.003 ; r=0.136, p=0.026$; and $r=0.169$, $p=0.011$, respectively). There were correlations between utilization of medication for depression and depression scores at baseline, visit 6 , and visit $12(r=0.231, p<0.001$; $r=0.334, p<0.001 ; r=0.227, p<0.001$, respectively). There was no association between SCOPA-AUT scores and utilization of medications that lower blood pressure at baseline, visit 6 , or visit 12 .

Longitudinal changes in SCOPA-AUT scores from baseline significantly predicted worsening in $\mathrm{S} \& \mathrm{E}$ scores at visit 6 (24 months) $(\beta=-0.21, p=0.015)$ and at visit 12 (60 months) $(\beta=-0.40, p=0.0001)$, after adjusting for age. Changes in the SCOPA-AUT score further predicted worsening in GDS score at visit $6(\beta=0.10, p<0.001)$ and at visit $12(\beta=0.08, p=0.012)$, but theydid not predict changes in MoCA or MDS-UPDRS part III scores (Table 2).

To understand whether depression medication usage confounds the relationship between longitudinal change in SCOPA-AUT scores and longitudinal change in ADLs scores, the regression analysis was repeated controlling for depression medication usage. The results at visit
B

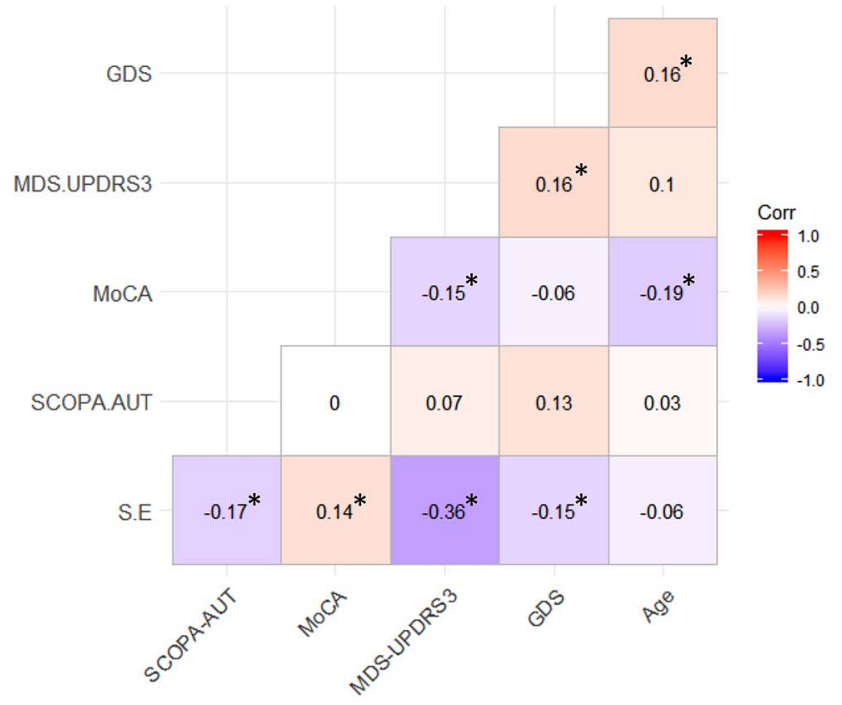

Scale, MDS-UPDRS3 Movement Disorders Society Unified Parkinson's Disease Rating Scale part III (motor examination), MoCA Montreal Cognitive Assessment scale, SCOPA-AUTScale for Outcomes in Parkinson's disease for Autonomic Symptoms, S ESchwab and England Activities of Daily Living scale

Table 2 Effects of longitudinal changes in Scale for Outcomes in Parkinson's disease for Autonomic Symptoms scores on clinical variables

\begin{tabular}{|c|c|c|c|c|}
\hline \multirow[t]{2}{*}{ Clinical variables } & \multicolumn{2}{|c|}{$\begin{array}{l}\text { Visit } 6 \\
\text { (24 months) }\end{array}$} & \multicolumn{2}{|c|}{$\begin{array}{l}\text { Visit } 12 \\
\text { (60 months) }\end{array}$} \\
\hline & $\beta$ & $p$ value & $\beta$ & $p$ value \\
\hline S\&E ADLs score & -0.21 & 0.015 & -0.40 & $<0.001$ \\
\hline GDS score & 0.10 & $<0.001$ & 0.08 & 0.012 \\
\hline MoCA score & -0.06 & 0.068 & 0.00 & 0.976 \\
\hline MDS-UPDRS part III score & 0.17 & 0.115 & 0.12 & 0.345 \\
\hline
\end{tabular}

Values adjusted for subject age at baseline

$6(\beta=-0.200, p=0.018)$ and at visit $12(\beta=-0.39$, $p=0.0002$ ) were comparable.

In a subanalysis, we investigated the impact of longitudinal changes in SCOPA-AUT subscores on decline in S\&E scores. Mean SCOPA-AUT subscores and prevalence of symptoms at each visit are presented in Electronic Supplementary Material (ESM) Table 1; these are similar to previous findings in this cohort [20]. We found that an increase in the cardiovascular subscore of SCOPA-AUT was the strongest predictor of a decline in S\&E score at visit $6(\beta=-0.92, p=0.031)$ and in fact was the only subscore that reached statistical significance. There were no significant SCOPA-AUT subscore predictors of ADLs function at visit 12 (see ESM Table 2). The 
SCOPA-AUT cardiovascular subscore was similarly the strongest predictor of an increase in GDS score at visit 6 $(\beta=0.49, p<0.001)$ and visit $12(\beta=0.38, p=0.024)$ (see ESM Table 3 ). There were no significant changes in thermoregulatory, pupilomotor, or sexual subscores between the baseline visit, visit 6 , or visit 12 .

We used mediation analysis, with bootstrapping-based assessment of confidence intervals, to investigate whether the relationship between autonomic symptom burden and ADLs function is mediated by depressive symptoms (Fig. 2). This analysis revealed a significant indirect effect of SCOPA-AUT scores on S\&E scores through the GDS scores at visit $12(\beta=-0.064,95 \% \mathrm{CI}-0.181$ to $-0.001)$ but not at visit $6(\beta=-0.033,95 \% \mathrm{CI}-0.083$ to 0.007 ) (Fig. 2), indicating that the effects of autonomic symptoms on functional decline are partially mediated by their contribution to depressive symptoms. Similar to the analysis reported above, the indirect effect of the cardiovascular subscore of SCOPA-AUT was a strong predictor of decline in ADLs at visit $12(\beta=-0.407,95 \%$ $\mathrm{CI}-0.946$ to -0.064$)$ but not at visit $6(\beta=-0.177$, $95 \%$ CI -0.444 to -0.043$)$. We further tested whether this mediation model was moderated by the severity of motor symptoms, as measured by the MDS-UPDRS part III, but this moderated mediation was not significant at visit 6 or visit 12 .

In a secondary analysis, we explored the associations between the presence of $\mathrm{OH}$ and other clinical variables. In total, there were 42 study participants with $\mathrm{OH}$ at baseline (13.4\%), 49 at visit $6(18.7 \%)$, and 42 at visit 12 $(18.58 \%)$. We found no significant association between the presence of $\mathrm{OH}$ and SCOPA-AUT scores at baseline, but there was a significant correlation between these two variables at visit 6 and visit $12(r=0.16, p=0.008$; $r=0.26, p<0.001$, respectively). Associations between presence of $\mathrm{OH}$ and other variables are presented in ESM Table 4.

A

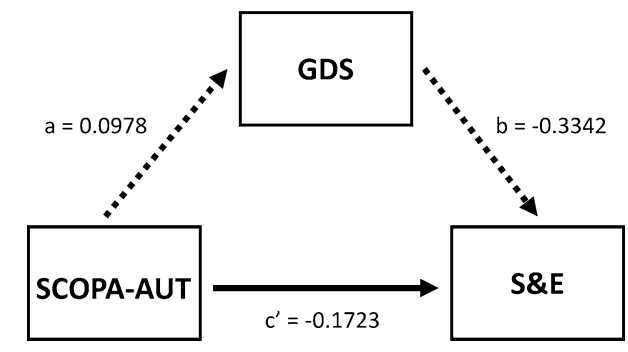

Fig. 2 Depressive symptoms mediate the longitudinal effects of autonomic symptoms on activities of daily living (ADLs) function at visit 12 but not at visit 6 . a Visit 6 , b visit 12 . Path $a$ indicates the direct effect of autonomic symptoms on depressive symptoms. Path $b$ indi-

\section{Discussion}

Our main finding is that longitudinal changes in autonomic symptom burden are associated with a worsening in ADLs in patients with early stage PD over an extended period of time. Few prior studies have investigated this relationship, and none over the time period included in this study. Although associations between autonomic symptoms and depression, and autonomic symptoms and ADLs, have been previously shown, the mediating effect of depression on the association between longitudinal changes in autonomic symptoms and ADLs had not been investigated before. In this regard we found that the negative impact of autonomic symptoms on function in PD is partially due to the impact of autonomic symptoms on depressive symptoms.

There have been few prior studies investigating longitudinal changes in autonomic symptoms and their impact on outcomes in persons with PD $[9,10]$. Visser et al. found that though baseline autonomic symptoms seemed to impact decline in health-related quality of life (HR-QOL), changes in autonomic symptom burden over 2 years did not seem to have a significant impact on this variable [9]. In their study, the average disease duration of participants at baseline was $>10$ years (standard deviation 6.5 years), and the mean change in the SCOPA-AUT score over the 2-year time period of the study was very small (1.1 points) [9], smaller than the change found in the current study. The lack of a significant result in this study may be due to these differences in variability [9]. Furthermore, it is likely that decline in HR-QOL is impacted by changes in SCOPA-AUT differently in different stages of PD; thus, the effects of changing SCOPA-AUT scores on HR-QOL were likely masked by the heterogeneity of this cohort with respect to disease duration. In another study, Merola et al. found a significant impact of change in SCOPA-AUT score and decline in ADLs score over a time period of 1 year, with gastrointestinal symptom change and cardiovascular symptom change in particular

B

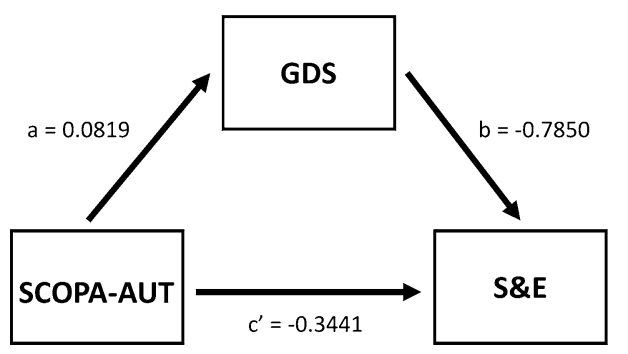

cates the direct effect of depressive symptoms on ADLs function. Path $c^{\prime}$ indicates the indirect effect of autonomic symptoms on ADLs function. $S \& E$ Schwab and England Activities of Daily Living scale 
being the primary drivers of this association [10]; this result supports our findings. This study had similar heterogeneity in the cohort with regards to disease duration as that of Visser et al. [9], with a mean and range in disease duration of $>10$ years and 1-26 years, respectively, and patients were followed for 12 months [10]. Though disease duration was adjusted for, there may have been limited SCOPA-AUT change over that time period to mask the association. The novelty of the current study is that the investigation was conducted in a homogenous cohort with regards to disease duration, i.e., early stage of PD (within 2 years of diagnosis), over a much longer period of time. Our study adds to the existing literature by demonstrating the significant impact of changes in autonomic symptom burden on decline in ADLs scores in early PD.

In a subanalysis, we investigated the associations between the presence of $\mathrm{OH}$ and other clinical variables. We found statistically significant associations between the presence of $\mathrm{OH}$ and SCOPA-AUT and ADL scores at visit 6, and between presence of $\mathrm{OH}$ and SCOPA-AUT and GDS scores at visit 12 . There were no statistically significant associations between $\mathrm{OH}$ and ADL scores, SCOPA-AUT scores, or GDS scores at baseline. The inconsistency in these results may be due to insufficient power of the study. $\mathrm{OH}$ is uncommon in the early stages of PD, which is reflected in the low frequencies observed in this cohort.

Our study again demonstrates the association between depression and decline in ADLs function, which has been investigated previously. However few studies have investigated the role of longitudinal changes in depressive symptoms on ADLs function or quality of life [5, 11-13]. Our study additionally found that longitudinal changes in depression act as a partial mediator in the association between changes in autonomic symptoms and decline in ADLs. Thus, we propose a model in which both autonomic symptoms and depression have direct impacts on ADLs function, in addition to the indirect mediation effect (Fig. 2). This finding indicates that depression should not be treated independently of autonomic symptoms in persons with PD.

Autonomic dysfunction has previously been implicated in the development of depression PD [21]. In support of the mediation effects found in the current study, it is likely that depression and autonomic dysfunction in PD, particularly cardiovascular autonomic dysfunction, share a common neurochemical substrate. Depression in PD patients may be attributable to a loss of dopaminergic and noradrenergic innervation in the brain [22], and treatment of depression in these presons may be more effective with agents that also block norepinephrine re-uptake [23]. Similarly, noradrenergic failure has been found to correlate with cardiovascular autonomic dysfunction in PD [24]. Distinct neural substrates of autonomic dysfunction in PD, particularly the hypothalamus, are being identified $[25,26]$, which includes structures with functions overlapping with depression networks [27, 28]. Noradrenergic dysfunction has also been implicated in REM sleep behavior disorder and cognitive decline in PD patients [29, 30]. Clustering of these symptoms may indicate the existence of a "malignant phenotype" in PD [30].

Controlling for depression medication usage in the association between longitudinal changes in autonomic symptom burden and longitudinal changes in ADLs scores did not significantly alter this relationship. However, we did find significant associations between severity of depressive symptoms and utilization of medications for depression. It is unclear whether this variable simply reflects the severity of depression rather than being an independent influence on autonomic symptom burden. Future prospective studies may be better able to differentiate these effects.

A limitation of this study is that objective measures of autonomic dysfunction, apart from orthostatic vital signs, were not available in this dataset. Previous studies have found erratic correlations between subjective autonomic symptom questionnaire scores and physiologic autonomic function testing results [31], though both subjective and objective autonomic symptoms correlate with disease severity [31]. Conversely, a correlation between abnormal autonomic testing results and frequency of autonomic symptoms in PD has been demonstrated [32]. Despite there being a lack of consensus regarding correlations between autonomic symptoms and autonomic function in PD, both subjective and objective measures of autonomic dysfunction in PD appear to have a negative impact on ADL function [33]. In the current study, associations were identified between autonomic symptom burden and the presence of orthostatic hypotension at visit 6 and visit 12, but not at baseline, though the lack of power in this analysis limits formal conclusions.

The importance of investigating longitudinal changes in clinical factors, as opposed to cross-sectional data, and their impact on decline in ADLs in PD patients is that there emerges a role for intervention. Baseline characteristics are dependent on the point in time when those data are collected and are, therefore, less meaningful with regards to a role for intervention. In this study, we chose to focus on intervenable characteristics, such as changes in autonomic symptom burden and depression, rather than on non-intervenable characteristics, such as age and disease duration, as our variables of interest. Specifically, it appears that cardiovascular autonomic symptoms are a major driver of this association. It is unclear whether interventions to improve autonomic symptoms in PD would improve outcomes; this would be an important area for further research. Furthermore, the impact of treating autonomic symptoms on the severity of depressive symptoms, and vice versa, deserve further examination. Future studies would benefit from evaluating both subjective and objective measures of autonomic dysfunction, as well 
as incorporating outcome measures evaluating quality of life in persons with PD.

Acknowledgements Data were obtained from the Parkinson's Progression Markers Initiative (PPMI) database. For up-to-date information on the study, visit www.ppmi-info.org. PPMI, a public-private partnership, is funded by the Michael J. Fox Foundation for Parkinson's Research and funding partners, including Abbvie, Avid Radiopharmaceuticals, Biogen, Bristol-Myers Squibb, Covance, GE healthcare, Genentech, GlaxoSmithKline, Lilly, Lundbeck, Merck, Meso Scale Discovery, Pfizer, Piramal, Roche, Servier, and UCB.

Author contributions Conceptualization: MS, ED; methodology: MS, C-HS, NB, J-AP, MS, ED; formal analysis and investigation: MS, C-HS, ED; writing — original draft preparation: MS; writing — review and editing: MS, C-HS, NB, J-AP, MS, ED; supervision: ED. All authors have approved the final article.

Funding The corresponding author, Miriam Sklerov, receives research support from the UNC Soo Neuroimaging research fund, as well as NC TraCS (UL1TR002489 Grant \# 550KR201815). All statistical analysis was carried out by Miriam Sklerov, Chia-Hao Shih, and Eran Dayan.

Data availability Any data not published within the article is available in a public repository.

\section{Compliance with ethical standards}

Conflict of interest Jose-Alberto Palma has been an advisory board member/consultant for Lundbeck, Biogen, PTC Therapeutics, and Dr. Reddy's Laboratories. He receives research funding from the NINDS, the Michael J. Fox Foundation, and the Familial Dysautonomia Foundation. He is the principal investigator in clinical trials funded by Biohaven and Theravance. Nina Browner has received Parkinson Foundation speakers and travel fees. Miriam Sklerov, Chia-Hao Shih, Styner, and Eran Dayan report no disclosures. This research did not receive any specific grant from funding agencies in the public, commercial, or not-for-profit sectors.

Ethical standards Data used in this manuscript were obtained from the Parkinson's Progression Markers Initiative (PPMI), a publicly available dataset. All study procedures were approved by the appropriate ethics committees at each participating site. All participants gave informed consent prior to inclusion in PPMI. For up-to-date information on the study, visit www.ppmi-info.org.

\section{References}

1. Jankovic J (2008) Parkinson's disease: clinical features and diagnosis. J Neurol Neurosurg Psychiatry 79:368-376. https://doi. org/10.1136/jnnp.2007.131045

2. Louis ED, Marder K, Cote L, Tang M, Mayeux R (1997) Mortality from Parkinson disease. Arch Neurol 54:260-264

3. Louis ED, Tang MX, Cote L, Alfaro B, Mejia H, Marder K (1999) Progression of parkinsonian signs in Parkinson disease. Arch Neurol 56:334-337

4. Alves G, Wentzel-Larsen T, Aarsland D, Larsen JP (2005) Progression of motor impairment and disability in Parkinson disease: a population-based study. Neurology 65:1436-1441. https://doi. org/10.1212/01.wnl.0000183359.50822.f2
5. Soh S-E, Morris ME, McGinley JL (2011) Determinants of health-related quality of life in Parkinson's disease: a systematic review. Parkinsonism Relat Disord 17:1-9. https://doi. org/10.1016/j.parkreldis.2010.08.012

6. De Pablo-Fernandez E, Tur C, Revesz T, Lees AJ, Holton JL, Warner TT (2017) Association of autonomic dysfunction with disease progression and survival in Parkinson disease. JAMA Neurol 74:970-976. https://doi.org/10.1001/jamaneurol .2017 .1125

7. Gray WK, Wood BH, Walker RW (2009) Do autonomic function tests in people with Parkinson's disease predict survival rates at 7 years follow-up? Mov Disord Off J Mov Disord Soc 24:24322434. https://doi.org/10.1002/mds.22834

8. Simuni T, Long JD, Caspell-Garcia C, Coffey CS, Lasch S, Tanner CM, Jennings D, Kieburtz KD, Marek K, Investigators PPMI (2016) Predictors of time to initiation of symptomatic therapy in early Parkinson's disease. Ann Clin Transl Neurol 3:482-494. https://doi.org/10.1002/acn3.317

9. Visser M, Verbaan D, van Rooden S, Marinus J, van Hilten J, Stiggelbout A (2009) A longitudinal evaluation of health-related quality of life of patients with Parkinson's disease, value health. J Int Soc Pharmacoecon Outcomes Res 12:392-396. https://doi. org/10.1111/j.1524-4733.2008.00430.x

10. Merola A, Romagnolo A, Rosso M, Suri R, Berndt Z, Maule S, Lopiano L, Espay AJ (2018) Autonomic dysfunction in Parkinson's disease: a prospective cohort study. Mov Disord Off J Mov Disord Soc 33:391-397. https://doi.org/10.1002/mds.27268

11. Marras C, McDermott MP, Rochon PA, Tanner CM, Naglie G, Lang AE (2008) Predictors of deterioration in health-related quality of life in Parkinson's disease: results from the DATATOP trial. Mov Disord Off J Mov Disord Soc 23(2008):653-659. https://doi. org/10.1002/mds.21853

12. Lawrence BJ, Gasson N, Kane R, Bucks RS, Loftus AM (2014) Activities of daily living, depression, and quality of life in Parkinson's disease. PLoS ONE 9:e102294. https://doi.org/10.1371/ journal.pone.0102294

13. Ravina B, Camicioli R, Como PG, Marsh L, Jankovic J, Weintraub D, Elm J (2007) The impact of depressive symptoms in early Parkinson disease. Neurology 69:342-347. https://doi. org/10.1212/01.wnl.0000268695.63392.10

14. Initiative PPM (2011) The Parkinson progression marker initiative (PPMI). Prog Neurobiol 95:629-635. https://doi.org/10.1016/j. pneurobio.2011.09.005

15. Visser M, Marinus J, Stiggelbout AM, Van Hilten JJ (2004) Assessment of autonomic dysfunction in Parkinson's disease: the SCOPA-AUT. Mov Disord Off J Mov Disord Soc 19:1306-1312. https://doi.org/10.1002/mds.20153

16. Goetz CG, Tilley BC, Shaftman SR, Stebbins GT, Fahn S, Martinez-Martin P, Poewe W, Sampaio C, Stern MB, Dodel R, Dubois B, Holloway R, Jankovic J, Kulisevsky J, Lang AE, Lees A, Leurgans S, LeWitt PA, Nyenhuis D, Olanow CW, Rascol O, Schrag A, Teresi JA, van Hilten JJ, LaPelle N (2008) Movement Disorder Society UPDRS Revision Task Force, Movement Disorder Society-sponsored revision of the Unified Parkinson's Disease Rating Scale (MDS-UPDRS): scale presentation and clinimetric testing results. Mov Disord Off J Mov Disord Soc 23:2129-2170. https ://doi.org/10.1002/mds.22340

17. Yesavage JA, Brink TL, Rose TL, Lum O, Huang V, Adey M, Leirer VO (1982) Development and validation of a geriatric depression screening scale: a preliminary report. J Psychiatr Res 17:37-49

18. Nasreddine ZS, Phillips NA, Bédirian V, Charbonneau S, Whitehead V, Collin I, Cummings JL, Chertkow H (2005) The Montreal cognitive assessment, MoCA: a brief screening tool for mild cognitive impairment. J Am Geriatr Soc 53:695-699. https://doi.org /10.1111/j.1532-5415.2005.53221.x 
19. Hayes AF (2017) Introduction to mediation, moderation, and conditional process analysis: a regression-based approach. Guilford Publications, New York

20. Stanković I, Petrović I, Pekmezović T, Marković V, Stojković T, Dragašević-Mišković N, Svetel M, Kostić V (2019) Longitudinal assessment of autonomic dysfunction in early Parkinson's disease. Parkinsonism Relat Disord 66:74-79. https://doi.org/10.1016/j. parkreldis.2019.07.008

21. Sagna A, Gallo JJ, Pontone GM (2014) Systematic review of factors associated with depression and anxiety disorders among older adults with Parkinson's disease. Parkinsonism Relat Disord 20:708-715. https://doi.org/10.1016/j.parkreldis.2014.03.020

22. Remy P, Doder M, Lees A, Turjanski N, Brooks D (2005) Depression in Parkinson's disease: loss of dopamine and noradrenaline innervation in the limbic system. Brain J Neurol 128:1314-1322. https://doi.org/10.1093/brain/awh445

23. Menza M, Dobkin RD, Marin H, Mark MH, Gara M, Buyske S, Bienfait K, Dicke A (2009) A controlled trial of antidepressants in patients with Parkinson disease and depression. Neurology 72:886-892. https://doi.org/10.1212/01.wnl.0000336340.89821 . $\mathrm{b} 3$

24. Sharabi Y, Goldstein DS (2011) Mechanisms of orthostatic hypotension and supine hypertension in Parkinson disease. J Neurol Sci 310:123-128. https://doi.org/10.1016/j.jns.2011.06.047

25. Dayan E, Sklerov M, Browner N (2018) Disrupted hypothalamic functional connectivity in patients with PD and autonomic dysfunction. Neurology 90:e2051-e2058. https://doi.org/10.1212/ WNL.0000000000005641

26. Sklerov M, Dayan E, Browner N (2018) Functional neuroimaging of the central autonomic network: recent developments and clinical implications. Clin Auton Res Off J Clin Auton Res Soc. https ://doi.org/10.1007/s10286-018-0577-0

27. Gerritsen L, Comijs HC, van der Graaf Y, Knoops AJG, Penninx BWJH, Geerlings MI (2011) Depression, hypothalamic pituitary adrenal axis, and hippocampal and entorhinal cortex volumes-the SMART Medea study. Biol Psychiatry 70:373-380. https://doi. org/10.1016/j.biopsych.2011.01.029

28. Lorenzetti V, Allen NB, Fornito A, Yücel M (2009) Structural brain abnormalities in major depressive disorder: a selective review of recent MRI studies. J Affect Disord 117:1-17. https:// doi.org/10.1016/j.jad.2008.11.021

29. Sommerauer M, Fedorova TD, Hansen AK, Knudsen K, Otto M, Jeppesen J, Frederiksen Y, Blicher JU, Geday J, Nahimi A, Damholdt MF, Brooks DJ, Borghammer P (2018) Evaluation of the noradrenergic system in Parkinson's disease: an 11C-MeNER PET and neuromelanin MRI study. Brain J Neurol 141:496-504. https ://doi.org/10.1093/brain/awx348

30. Pilotto A, Romagnolo A, Tuazon JA, Vizcarra JA, Marsili L, Zibetti M, Rosso M, Rodriguez-Porcel F, Borroni B, Rizzetti MC, Rossi C, Vizcarra-Escobar D, Molano JR, Lopiano L, Ceravolo R, Masellis M, Espay AJ, Padovani A, Merola A (2019) Orthostatic hypotension and REM sleep behaviour disorder: impact on clinical outcomes in $\alpha$-synucleinopathies. J Neurol Neurosurg Psychiatry 90:1257-1263. https://doi.org/10.1136/jnnp-2019-320846

31. Oh ES, Lee JH, Seo JG, Sohn EH, Lee AY (2011) Autonomic and cognitive functions in Parkinson's disease (PD). Arch Gerontol Geriatr 52:84-88. https://doi.org/10.1016/j.archger.2010.02.005

32. Magerkurth C, Schnitzer R, Braune S (2005) Symptoms of autonomic failure in Parkinson's disease: prevalence and impact on daily life. Clin Auton Res Off J Clin Auton Res Soc 15:76-82. https://doi.org/10.1007/s10286-005-0253-z

33. Merola A, Romagnolo A, Rosso M, Lopez-Castellanos JR, Wissel BD, Larkin S, Bernardini A, Zibetti M, Maule S, Lopiano L, Espay AJ (2016) Orthostatic hypotension in Parkinson's disease: does it matter if asymptomatic? Parkinsonism Relat Disord 33:65-71. https://doi.org/10.1016/j.parkreldis.2016.09.013 